\title{
STEM-based analysis of functional defects in ferroelectric ErMnO3
}

Antonius T. J. van Helvoort ${ }^{1}$, Aleksander Mosberg ${ }^{2}$, Ursula Ludacka ${ }^{1}$, Theodor S. Holstad ${ }^{3}$, Donald M. Evans $^{4}$ and Dennis Meier ${ }^{1}$

${ }^{1}$ NTNU, United States, ${ }^{2}$ SuperSTEM, Daresbury, England, United Kingdom, ${ }^{3}$ DTU, United States, ${ }^{4}$ University of Augsburg, United States

Ferroelectric materials, 100 years after their discovery, still intrigue. Recently, ferroelectrics with charged domain walls have come into the spotlight, holding great potential as functional entities in future nanoelectronic devices [1]. A critical aspect of tuning complementary properties to realize such devices is achieving control over point defects in the ferroelectric materials. In this contribution, hexagonal ErMnO3, a model system for improper ferroelectrics with charged domain walls, was modified at the tens of nm-scale by an electric field from conductive atomic force microscopy [2]. We demonstrate that combining a broad set of transmission electron microscopy techniques is essential for developing a fundamental understanding of the nature of emergent electric-field driven modifications and related phenomena, enabling new nanotechnology applications of ferroelectric materials.

For hexagonal ErMnO3, the polarization direction can be deduced from lattice imaging in the 100direction by considering the relative positions of the Er-columns via aberration-corrected high-angle annular dark-field scanning transmission electron microscopy (HAADF-STEM) [3]. However, lattice defects and changes in composition can lead to variations in the net polarization. To quantify such variations, a detailed analysis of the relative Er-column positions is required. Here we used serial HAADFSTEM imaging with short frame times and consecutive image reconstruction. Compared to single frame imaging, scan distortions can be strongly reduced by using the SmartAlign approach, reconstructing an image based on the total scanning series [4]. The relative column positions in the final image were analyzed using the open-source package Atomap [5]. We show that with a statistical analysis differences in the Er-plane spacings in the $c$-direction can be achieved with a sub $10 \mathrm{pm}$ precision. Thereby, high spatial resolution polarization studies can be realized. The results demonstrate that the ferroelectric order parameter is essentially unchanged in the modified volume.

However, we found that point defects have formed within the modified region. This can be studied by electron energy loss spectroscopy (EELS), combined with structure modelling and spectrum simulations. The nature of these defects can be deduced by studying subtle variations in the O-K fine structure and the $\mathrm{Mn}$ L-edge between the bulk and modified $\mathrm{ErMnO}_{3}$ areas. Our analysis point toward electric-field induced anti-Frenkel defects, i.e., charge-neutral oxygen interstitial-vacancy pairs, within the modified region [2]. The results from fine structure analysis helped to test several possible explanations for the observed increased relative conductivity in the modified areas. An important prerequisite for such sensitive studies is pristine and site-specific specimen preparation.

Our results demonstrate the importance of pushing lattice imaging and more in-depth EELS studies to unravel the complex structure of ferroelectric oxides and the impact of point defects on their functional properties. In recent years, 4D STEM in combination with greatly improved detector technology has become the state-of-the-art for high spatial resolution analysis in STEM. These methods led to breakthroughs in understanding materials on the atomic scale, including detecting defects embedded in functional oxides [6]. Here the novel approach could reveal more details of the created defect structure. 
Considering the study of modified $\mathrm{ErMnO}_{3}$, both point defects as well as lattice displacement affecting the polarization must be considered. They could be present simultaneously, hence their signatures must be disentangled. Both of the structural characteristics are main driving forces for tailoring the electronic properties [7].

\section{References}

1: G. F. Nataf, et al., Nat. Rev. Phys. 2, 634 (2020)

2: D. M. Evans, et al., Nat. Mat., 9, 1 (2020).

3: M. Han, et al., Microsc. Microanal., 18, 1358 (2012).

4: L. Jones, et al., Adv. Struct. Chem. Imaging, 1, 8 (2015).

5: M. Nord, et al., Adv. Struct. Chem. Imaging, 3, 9 (2017).

6: C. Ophus, Microsc. Microanal. 25, 563 (2019)

7: Acknowledgements: The Research Council of Norway is acknowledged for support to the Norwegian Micro- and Nano-Fabrication Facility, NorFab (245963/F50) and Norwegian Centre for Transmission Electron Microscopy, NORTEM (197405). A.B.M. was supported by NTNU's Enabling technologies: Nanotechnology. D.M. thanks NTNU for support through the Onsager Fellowship Programme and NTNU Stjerneprogrammet. 